\title{
A Global COVID-19 Pandemic Needs an Integrated Global Response
}

\begin{abstract}
The global pandemic caused by coronavirus disease 2019 is challenging health care workers and public health specialists around the world. Most data and policy, including recommendations for controlling the pandemic, measures of lockdown, and strategy to relaxing lockdown, all appear to emerge from the high income countries. We present the situation in the UK, and consider the inequalities globally, calling to attention critical concerns faced by vulnerable groups. Ethnic minorities, migrants, those in care homes, those in mental health services, and health staff from migrant or minority groups are all at higher risk of death and need for intensive care. We call for more comprehensive evidence base to be generated across nations, and for the global health community to be more cognisant of actions and approaches in the Global South, and translating these lessons to develop more sustainable recovery pathways. Learning across geopolitical and economic divides and addressing entrenched social inequalities are critical if we are to recommence international travel and business, and ensure that health protections are enforced globally.
\end{abstract}

Keywords: Coronavirus disease 2019, Global North-South, inequalities, migrants, minorities

The coronavirus disease 2019 (COVID-19) pandemic is leading to very high levels of premature mortality in high- and low-income countries, albeit the levels, timing, and reporting vary by countries. Comparisons of crude death rates across countries do not accommodate different age and gender structures, nor variations in other risk factors such as social inequalities, population density, and demographic (e.g., age structure and at-risk populations), nor the background levels of mortality due to other communicable and noncommunicable disease, and capacity of health systems to respond. All of these influence mortality rates but tend to be omitted in the political rhetoric and claims of the crises "averted" by governments.

The WHO and national governments are rightly pursuing rapid research into potentially effective treatments, largely pharmacological agents, to limit harms of the virus and by repurposing known medications licensed for other uses, and considered safe to test. There is also a race on developing vaccinations and effective drugs, though with little considerations of how these public goods can be shared fairly

\begin{abstract}
Submission: $26-05-20$ Acceptance: 02-06-20

Decision: 29-05-20 Web Publication: $14-08-20$

This is an open access journal, and articles are distributed under the terms of the Creative Commons Attribution-NonCommercial-ShareAlike 4.0 License, which allows others to remix, tweak, and build upon the work non-commercially, as long as appropriate credit is given and the new creations are licensed under the identical terms.
\end{abstract}

For reprints contact: reprints@medknow.com within and between nations. There is a lack of attention to the wider social determinants and the capacity and actions of health systems that might amplify inequalities and leave those in greatest need without adequate care. A more silent narrative is of potentially varying levels of recognition and treatments and pathways to care for COVID-19 dependent on a number of health system characteristics. These include:

- Workforce availability and skills and capacity in primary and secondary care and in public health systems

- Health systems capacity to respond with adequate testing, tracing, and intensive care with relevant respiratory and cardiovascular condition management. Assessment of these capacities must account for the structure and organization of services, including casualization and the role of informal sector providers: charity sectors, lay health workers, and the wider social and cultural assets that reinforce with health systems

- Social inequalities create differential risks for different populations. Are there standard protocols across vastly different health and political contexts?

Who are high-risk groups? People with severe mental illnesses living in supported accommodation, or located on inpatient

How to cite this article: Bhui KS, Kapilashrami A. A Global Covid-19 Pandemic Needs an Integrated Global Response. World Soc Psychiatry 2020;2:84-7.
Kamaldeep S. Bhui ${ }^{1,2}$, Anuj Kapilashrami ${ }^{3}$

${ }^{1}$ Professor of Psychiatry, Department of Psychiatry, University of Oxford, Warneford Hospital, Oxford, ${ }^{2}$ Hon. Professor of Psychiatry, Queen Mary University of London, ${ }^{3}$ Centre for Global Public

Health, Institute of Population Health Sciences, Queen Mary University of London, London, England, UK
Address for correspondence: Prof. Kamaldeep S. Bhui, Department of Psychiatry, University of Oxford, Warneford Hospital, Oxford, OX3 7JX, UK. E-mail:kam.bhui@psych.ox.ac. $u k$

Access this article online Website: www.worldsocpsychiatry.org

DOI: 10.4103/WSP.WSP_49_20 Quick Response Code:

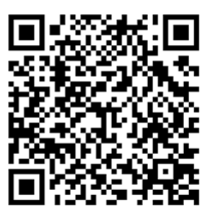


units, and those with comorbid conditions are all likely to be more susceptible to complex and worrisome outcomes of the infection. Furthermore, the short- and long-term social, psychological, and psychiatric sequelae are not being considered; these include the impacts of social isolation on physical and emotional well-being; the impacts of physical inactivity if movements are restricted; fears of contamination and obsessive-compulsive and anxiety disorders; and depressive reactions as well as complicated grief, given we are not able to mourn or attend funerals and comfort each other in usual ways.

Global health campaigns and movements highlight the disparities between the Global North and Global South - the levels of investment, the plights of citizens in these imagined jurisdictions, and prioritization (or neglect) of different health conditions (notably investment in mental health care is limited, stigmatized, and relies more on informal sectors). The Inverse Care Law applies generally and also in the instances of crisis and public health interventions. Thus, we would expect to see that those most in need may not receive the care and resources that can support them. Yet, low- and middle-income countries (LMICs) appear less party to commentary, new data and publications, political debate, and screening and testing to inform actions. Moreover, there is a marked reluctance to learn from efforts in LMICs or from previous experiences of tackling pandemics.

\section{High- and low-income countries: Social status and ethnic disparities}

In high-income countries, we are seeing higher rates of mortality among the already-marginalized groups. A key theme to emerge in the UK and USA is the racial or ethnic disparities in death rates and greater need for intensive care. ${ }^{[1,2]}$ The explanations are multiple, ranging from ethnic variations of biological risks such as ACE2 receptor variants, androgen receptor variants, higher risks of diabetes and hypertension, variations in immune mechanisms, obesity and inactivity, and healthy lifestyles and nutrition. ${ }^{[3,4]}$ Preexisting ethnic differences in socioeconomic status, income, employment, healthy lifestyles, and exposure to racism and societal inequalities are also proposed as the fundamental causes of inequalities, that are simply exposed and brought to conscious awareness at this challenging time. ${ }^{[5-7]}$ The pandemic has reminded us of the stark underlying structural inequalities which disadvantage particular groups in the society. Indeed, ethnic differences in mortality due to pandemics are not new. ${ }^{[8-10]}$ Furthermore, ethnic minority health workers appear to be at greater risk of infection and death. Again, ethnic disparities are alarming, leading to calls for formal inquiries and public investigations and hearings. ${ }^{[1]]}$

A rapid series of analyses by all with rapid publications as preprints generate more uncertainty, for example, ACE2 variants may not be relevant (https://www.medrxiv.org/content/10.1101/202 0.05.01.20085860v1.article-info). Although ethnic disparities are real, they are attenuated only partially by adjusting for socioeconomic conditions and comorbidities, suggesting health-care capacity and practice may vary by ethnic group (https://www. medrxiv.org/content/10.1101/ 2020.05.06.20092999v1). Indeed, the notion of structural inequalities and racism in the society leading to health disparities is well established in evidence, and racism even in health-care practices and recruitment and training of doctors continues to be lamented (https://discoversociety. org/2020/04/17/racism-is-the-root-cause-of-ethnic-inequities -in-covid19/. ${ }^{[12-14]}$

What is the global view of this? Are LMICs seeing a similar effect?

Both the biological and socioeconomic explanations, if valid, have implications for LMICs facing more extremes: health systems capacity, poverty, poor housing and crowded conditions, poorer nutrition, and a shortage of investment in health care. In LMICs, the crisis will hit vulnerable populations even harder. In India, for example, the sudden lockdown left 400 million workers out of work and at the brink of starvation and impoverishment. ${ }^{[15]}$ Yet, assessment of how the outbreak maps onto social inequalities (based on caste, SES, livelihoods, and indigeneity) is almost absent from the COVID-19 discourse on Global South, apart from death rate comparisons. With the exceptions of a few commentaries of the plight of migrants and refugees in some countries in South Asia and Southern Africa, there are hostile alerts about and shaming of particular minority groups (religious communities and faith groups), and analysis of the distribution of risks is lacking.

\section{Health impact and responses}

The COVID-19 pandemic has also led to lockdown, disruption, even in high-income countries, of usual health services, including mental health care. Apart from social isolation and anxiety and worsening of the existing mental illnesses, ${ }^{[16]}$ in the UK, there are proposals, for example, for mental health act protections to be reduced and periods of detention in crisis to be extended (https://synergicollaborativecentre.co.uk/newcoronavirus-act-may-increase-the-mental-health-detention-ofethnic-minority-people/).

World Social Psychiatry rightly shines a light on the global state of affairs. We need more research and open discussion about the public health and mental health impacts of the lockdown, include the consequences of restrictions on accessing health services, which are already limited in low-income countries. We need to assess adherence to preventive advice and access to testing and health interventions.

All-cause mortality is proposed as the only real indicator that can be compared across countries, yet this involves delays in securing accurate data for deaths in all settings and across very different traditions and systems of recording and data 
collection. The more muted patterns of COVID-19 mortality burden among LMICs have been attributed to a number of factors including demographic, stage of pandemic, data quality, and reporting, including the likelihood of not investigating, recording, or attributing deaths outside health systems (i.e., in the community) to COVID-19. The true impact of COVID-19 will be more long lasting and difficult to disentangle. The burden on economies and societies will almost certainly be greater in the Global South; yet the evidence and analysis, and responses, now and in future, will be grossly insufficient unless local systems of tracking can be developed and analyses and actions can be modelled for their local scenarios. While countries launch preventive measures and prepare health systems for the marathon that awaits, it is important to remind ourselves that the world must tap into the accumulated knowledge base LMICs have gained in combating prior outbreaks (e.g., successful community testing and tracking methods in Kerala, efficient use of digital tools in South Africa).

It is timely that as public health academics, we pose important questions and collectively define a research agenda, calling for greater attention to global and national inequalities in off-setting the impact of the pandemic. As a starting point, we outline five key issues:

- How might socioeconomic and fundamental causes be tackled around the globe - high-income countries have failed for decades to address this; can we find better solutions in the LMICs?

- What impact will lockdown and the inevitable economic strains and unemployment have on the rates of mental illness and care?

- How can digital technology buffer these effects, and be most exploited for public good in LMICs?

- How does accumulated disadvantage experienced by ethnic minorities and migrants in high-income countries LMICs where the levels of poverty are extremely harsh and care provision is already limited, especially for mental health care?

- How will health investments rise to tackle the many sequelae and chronic conditions of COVID-19 to reflect this evenly across the globe?

There is no better time to call for more integrated, low-cost, and adaptive interventions and investment for health care in LMICs, more intensive actions to tackle the social determinants of poor health (physical and mental), and fundamental causes of disparities which are shaped by the social and political conditions of each nation state and their regional geopolitical economies.

\section{Further Reading}

\section{BLOGS}

https://www.dw.com/en/covid-19-death-rate-sinking-datareveals-a-complex-reality/a-53365771

https://discoversociety.org/2020/04/17/racism-is-the-root- cause-of-ethnic-inequities-in-covid19

https://www.cdc.gov/coronavirus/2019-ncov/need-extraprecautions/racial-ethnic-minorities.html

https://ghpu.sps.ed.ac.uk/wp-content/uploads/2020/04/ Qureshi-Kasstan-Meer-Hill_working-paper_COVID19ethnic-minorities_240420.pdf

https://synergicollaborativecentre.co.uk/new-coronavirusact-may-increase-the-mental-health-detention-of-ethnicminority-people/

Pre prints

https://www.medrxiv.org/content/10.1101/2020.05.02.20078 642v1.full.pdf

https://www.medrxiv.org/content/10.1101/2020.04.25.20079 491v1.full.pdf

https://europepmc.org/article/ppr/ppr158187

https://www.medrxiv.org/content/10.1101/2020.05.06.20092 999v1

https://www.medrxiv.org/content/10.1101/20200.05.01.2008 $5860 \mathrm{v} 1$.article-info

\section{Financial support and sponsorship}

Nil.

\section{Conflicts of interest}

There are no conflicts of interest.

\section{REFERENCES}

1. Centre I.C.N.A.a.R., ICNARC Report on COVID-19 in Critical Care 2019. Available from: https://www.icnarc.org/Our-Audit/ Audits/Cmp/Reports [Last accessed on 2020 Jul 18].

2. Laurencin CT, McClinton A. The COVID-19 pandemic: A call to action to identify and address racial and ethnic disparities. J Racial Ethn Health Disparities 2020.

3. Khunti K, Singh AK, Pareek M, Hanif W. Is ethnicity linked to incidence or outcomes of covid-19? BMJ 2020;369:m1548.

4. McCoy J, Wambier CG, Vano-Galvan S, Shapiro J, Sinclair R, Müller Ramos $\mathrm{P}$, et al. Racial variations in COVID-19 deaths may be due to androgen receptor genetic variants associated with prostate cancer and androgenetic alopecia. Are anti-androgens a potential treatment for COVID-19? J Cosmet Dermatol 2020;19:1542-3

5. Kapilashrami A, Bhui K. Mental health and COVID-19: Is the virus racist? Br J Psychiatry 2020:1-3.

6. Hankivsky O, Kapilashrami A. Beyond Sex and Gender Analysis: An Intersectional View of the COVID-19 Pandemic Outbreak and Response. Global Policy Institute, QMUL; 2020. Available from: https://www.qmul.ac.uk/media/global-policyinstitute/Policy-brief-COVID-19-and-intersectionality.pdf [Last accessed on 2020 Jul 18].

7. Laster Pirtle, W. N. (2020). Racial Capitalism: A Fundamental Cause of Novel Coronavirus (COVID-19) Pandemic Inequities in the United States. Health Education \& Behavior. https://doi. org/10.1177/1090198120922942.

8. Zhao H, Harris RJ, Ellis J, Pebody RG. Ethnicity, deprivation and mortality due to 2009 pandemic influenza A (H1N1) in England 
during the 2009/2010 pandemic and the first post-pandemic season. Epidemiol Infect 2015;143:3375-83.

9. Placzek H, Madoff L. Effect of race/ethnicity and socioeconomic status on pandemic H1N1-related outcomes in Massachusetts. Am J Public Health 2014;104:e31-8.

10. Dee DL, Bensyl DM, Gindler J, Truman BI, Allen BG, D'Mello T, et al. Racial and ethnic disparities in hospitalizations and deaths associated with 2009 pandemic Influenza A (H1N1) virus infections in the United States. Ann Epidemiol 2011;21:623-30.

11. Rimmer A. Covid-19: Disproportionate impact on ethnic minority healthcare workers will be explored by government. BMJ 2020;369:m1562.

12. Salway S, Holman D, Lee C, McGowan V, Ben-Shlomo Y, Saxena S, et al. Transforming the health system for the UK's multiethnic population. BMJ 2020;368:m268.

13. Mathew R. Rammya Mathew: Racism in medicine-migrant doctors aren’t here just to "fill a gap". BMJ 2020;368:m483.
14. Adebowale V, Rao M. Racism in medicine: Why equality matters to everyone. BMJ 2020;368:m530.

15 Kapilashrami A, on behalf of the Migration Health South Asia network. 2020. Neglect of low-income migrants in covid-19 response. A South Asian perspective on the failures of global and national public health policies. Available from: https://blogs.bmj. com/bmj/2020/05/29/neglect-of-low-income-migrants-in-covid19-response/ [Last accessed on $2020 \mathrm{Jul} 18]$.

16. Holmes EA, O’Connor RC, Perry VH, Tracey I, Wessely S, Arseneault L, et al. Multidisciplinary research priorities for the COVID-19 pandemic: A call for action for mental health science. Lancet Psychiatry 2020;7:547-60. 